\title{
How digital tools can advance quality and equity in genomic medicine
}

Yvonne Bombard (iD ${ }^{1,2} \bowtie$ and Robin Z. Hayeems (iD ${ }^{2,3}$

Now more than ever, digital applications are essential to accessing genetics services and optimizing their delivery. At this watershed moment, digital solutions must be balanced with the merits of human interaction, without compromising quality or exacerbating existing genomic and technological disparities.

digital
solutions can
reduce disparities
in access ... and
engage broader
communities
of health-care
providers

${ }^{1}$ Genomics Health Services Research Program, Li Ka Shing Knowledge Institute, St. Michael's Hospital, Unity Health, Toronto, ON, Canada.

2Institute of Health Policy Management and Evaluation, University of Toronto, Toronto, ON, Canada.

${ }^{3}$ Program in Child Health Evaluative Sciences, The Hospital for Sick Children, Toronto, ON, Canada.

凶e-mail:yvonne.bombard@ utoronto.ca

https://doi.org/10.1038/ s41576-020-0260-x
COVID-19 has launched our community of practice into a new reality of digital care. On top of pre-existing wait times and workforce shortages in clinical genetics ${ }^{1,2}$, the pandemic has revealed the urgency of virtual care interventions to minimize interruptions and compromised access to care. Digital health applications, portals and platforms are emerging across many medical disciplines, including genomic medicine. A scan of the clinical genetics landscape confirms the increasing use of chatbots, digital portals and machine learning algorithms to facilitate patient intake, phenotyping, counselling, laboratory analysis and result reporting (FIG. 1). These digital solutions have become essential to enable access to basic genetics services and optimize their delivery. For remote and less well-served communities, digital tools can improve access, reduce wait times, and enable continuity and quality of care. Patient portals in other areas have improved patient engagement, medication adherence, use of preventive services and clinical outcomes ${ }^{3}$. Digital decision support tools for patients and clinicians have broadened the reach and efficiency of genomic medicine $^{4}$ by enabling easier access to testing and counselling resources, especially for non-genetics specialists or those practising in resource-limited settings. By off-setting administrative burden on providers, digital intake forms and pedigree programmes can, in turn, increase the time available for meaningful, patient-centred consultations.

However, these profound clinical opportunities expose a myriad of technological divides for both providers and patients. From providers' perspectives, new digital solutions will require interoperability with existing laboratory information and medical record systems and will need to seamlessly integrate multiple data sources and formats to minimize cognitive data overload ${ }^{5}$. A model of care that depends on digitization will require substantial capacity building among practitioners to equip them with the required skillset. Similarly, optimizing patients' computer and health literacy as well as robust internet access while maintaining privacy is fundamental and likely to be challenging to achieve on a large scale. While reshaping the patient-provider interaction may alter relationship dynamics, whether this alteration proves favourable or unfavourable with respect to the patient experience remains to be seen.

\section{Shifting dynamics in care}

Early experiences leveraging telehealth capabilities under COVID-19 restrictions are signalling a shift in the dynamics of care. From advisory meetings with geneticists and genetic counsellors in tertiary-care settings, our data indicate that clinical geneticists detect a heightened sense of trust among patients receiving virtual care compared with in-person visits (Y.B. and R.Z.H., unpublished observations). Outside of a clinic setting, their perception is that the doctor-patient hierarchy dissipates, allowing the patient to feel more comfortable speaking and sharing ideas. They welcome emerging digital tools to support a range of clinical and administrative tasks (for example, phenotyping, family history taking, consent, education, referrals and integration of multi-disciplinary teams, and appointment booking). However, they feel strongly about retaining real-time engagement, be it in person or video conference-based, with patients to build rapport, understand the nuances of the patient and their circumstances, and ensure complex information is delivered with the expertise it requires. Both a commitment to patient-centred care and liability concerns underpin this perspective.

\section{Balancing digital access with human interaction} Ultimately, our community of practice will need to consider how to best balance the efficiencies granted by digital tools with the comfort and quality of in-person encounters. For example, despite patient enthusiasm for the Genomics ADvISER, a digital health application to guide patients' choices in the case of incidental findings, some patients still wanted a human touch ${ }^{6}$ - an obvious but critical gap in the increasing digitization of care. One potential solution is a hybrid model whereby in-person (or video conference-based) encounters are supplemented with digital pre- and post-test tools. To this end, we are developing the Genetics Navigator (FIG. 1), an innovative 


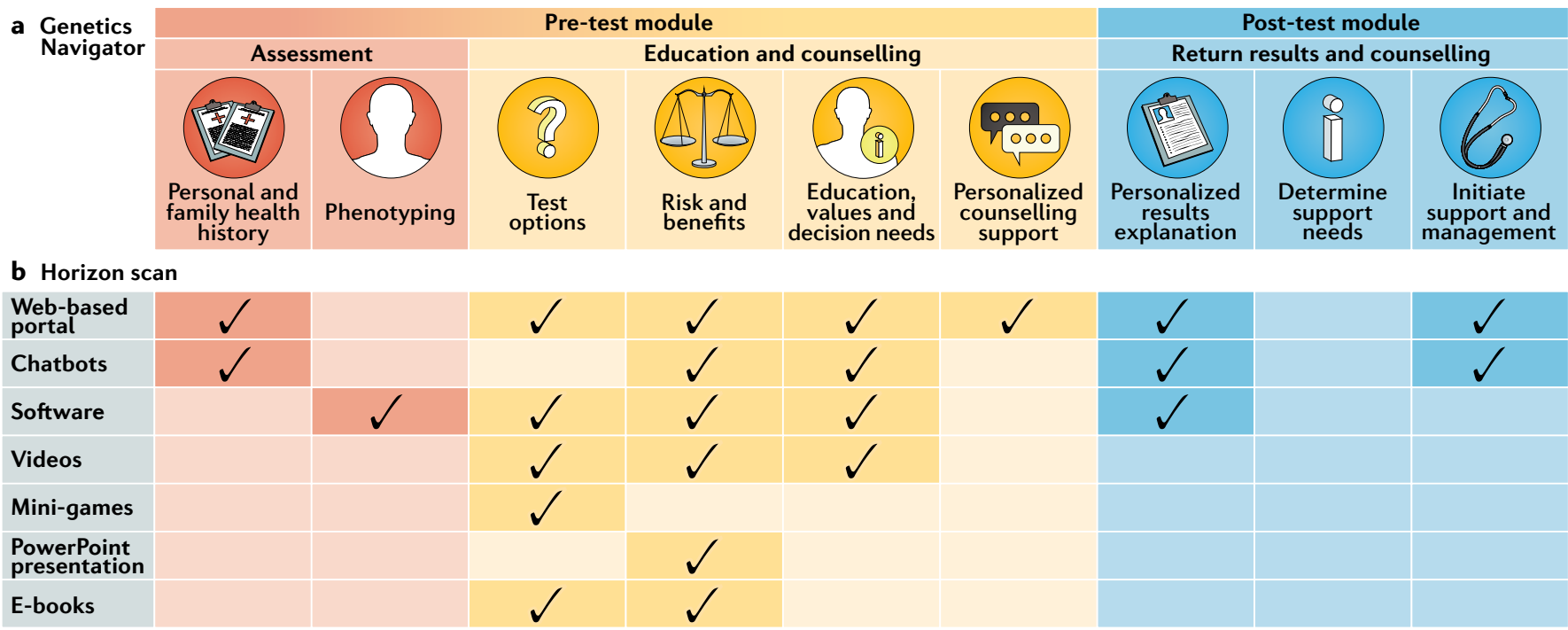

Fig. 1 | Digital care tools in genomic medicine. a | The genetics service pathway that will be delivered digitally by the Genetics Navigator, a digital application under development aimed at delivering the full spectrum of clinical services. b | Horizon scan of the modalities used in existing digital care tools for each phase of the genetics service pathway.

model of care for genomic medicine designed to facilitate and optimize the delivery of the entire genomic testing pathway. Unlike existing tools that focus on specific components of genomic service delivery (FIG. 1), the Genetics Navigator aims to supplement the full pathway of care, from pre-test counselling, education, decision support, laboratory reporting, personalized return of results, posttest counselling, customized management recommendations and referrals to automated recontact for reanalysis of variants over time. Aligned with the principles of user-centred design, the Genetics Navigator will include a chatbot and audio, visual and graphical components to engage patients and deliver quality, comprehensive care. While such automation may improve efficiencies and reduce provider burden, it remains to be determined with what role genetics providers will be comfortable as these models of care evolve.

\section{A watershed moment}

Our community of practice is at a watershed moment. The efficiencies of digital solutions have exposed technological divides that layer on top of existing disparities in access to care and representation in genomics. On the one hand, digital solutions can reduce disparities in access by deploying genomic services in diverse and underserved settings outside academic research centres and engage broader communities of health-care providers. On the other hand, the databases that we use to interpret variant pathogenicity suffer from long-standing biases because of the under-representation of genome data across ethnic groups ${ }^{7}$. The quality of care afforded by digital solutions is only as good as the data input into these systems. Existing biases may therefore be reinforced by digital solutions, disproportionately disadvantaging those already marginalized by genomic medicine ${ }^{8}$.

Similarly, there is a critical need to reconsider the proprietary nature of some digital tools and data access to avoid exacerbating existing genomic and technological disparities. Participatory governance models, where control is shared with patients, can not only increase patients' trust and address their privacy concerns but enable co-design of patient-centred solutions that ultimately improve patients' experiences and outcomes?.

We anticipate that comfort and efficiencies with digital genomic applications will evolve as we acquire more experience as well as the clinical and economic evidence to inform their adoption. The onus is now on our community to build the evidence base and required governance model to ensure equitable and sustainable digital genomic medicine.

1. Cooksey, J. A., Forte, G., Flanagan, P. A., Benkendorf, J. \& Blitzer, M. G. The medical genetics workforce: an analysis of clinical geneticist subgroups. Genet. Med. 8, 603-614 (2006).

2. Abacan, M. et al. The global state of the genetic counseling profession. Eur. J. Hum. Genet. 27, 183-197 (2019).

3. Dendere, R. et al. Patient portals facilitating engagement with inpatient electronic medical records: a systematic review. J. Med. Internet Res. 21, e12779 (2019).

4. Bombard, Y. et al. Effectiveness of the Genomics ADvISER decision aid for the selection of secondary findings from genomic sequencing a randomized clinical trial. Genet. Med. 22, 727-735 (2020).

5. Abernethy, A. P. et al. Rapid-learning system for cancer care. J. Clin. Oncol. 28, 4268-4274 (2010).

6. Bombard, Y. et al. The Genomics ADvISER: development and usability testing of a decision aid for the selection of incidental sequencing results. Eur. J. Hum. Genet. 26, 984-995 (2018).

. Manrai, A. K. et al. Genetic misdiagnoses and the potential for health disparities. N. Engl. J. Med. 375, 655-665 (2016).

8. Bombard, Y. \& Mighton, C. Recontacting clinical genetics patients with reclassified results: equity and policy challenges. Eur. J. Hum. Genet 27, 505-506 (2019).

9. McGuire, A. L. et al. Importance of participant-centricity and trust for a sustainable medical information commons. J. Law Med. Ethics 47, 12-20 (2019).

\section{Acknowledgements}

During the development of this work, Y.B. was supported by a CIHR New Investigator Award and R.Z.H. was supported by a SickKids Foundation/ CIHR New Investigator Award. The authors thank M. Clausen, S. Lucca and D. Abd Assamad for assistance with the horizon scan.

\section{Competing interests}

The authors declare no competing interests.

\section{RELATED LINKS}

Genomics ADvISER: www.genomicsadviser.com 